\title{
MAP Decoding of Correlated Sources over Soft-Decision Orthogonal Multiple Access Fading Channels with Memory
}

\author{
Seyed Parsa Beheshti \\ Email: s.p.beheshti@queensu.ca
}

\author{
Fady Alajaji \\ Email: fady@mast.queensu.ca
}

\author{
Tamás Linder \\ Email: linder@mast.queensu.ca
}

\begin{abstract}
We consider the joint source-channel coding (JSCC) problem where the real valued outputs of two correlated memoryless Gaussian sources are scalar quantized, bit assigned, and transmitted, without applying any error correcting code, over a multiple access channel (MAC) which consists of two orthogonal point-to-point time-correlated Rayleigh fading subchannels with soft-decision demodulation. At the receiver side, a joint sequence maximum a posteriori (MAP) detector is used to exploit the correlation between the two sources as well as the redundancy left in the quantizer's indices, the channel's softdecision outputs, and noise memory. The MAC's sub-channels are modeled via non-binary Markov noise discrete channels recently shown to effectively represent point-to-point fading channels. For the simple case of quantizing the sources with two levels, we establish a necessary and sufficient condition under which the joint sequence MAP decoder can be reduced to a simple instantaneous symbol-by-symbol decoder. Then, using numerical results obtained by system simulation, it is observed that when the sources are highly correlated and soft-decision quantization is used, JSCC can profit from a high correlation in the channel noise process and provide significant signal-to-distortion ratio improvements of up to $6.3 \mathrm{~dB}$ over a fully interleaved channel.
\end{abstract}

Index Terms-Joint source-channel coding, correlated Gaussian sources, multiple access channels, MAP decoding, channels with memory, time-correlated fading channels, scalar quantization.

\section{INTRODUCTION}

Joint source-channel coding (JSCC) has been shown to have significant advantages over Shannon's separate sourcechannel coding strategy [1] in practical communication systems with complexity and delay constraints [2], [3]. In [4], sequence maximum a posteriori (MAP) decoding is studied for a system with no algebraic channel coding and channel interleaving. This is one approach to design a JSCC system based on lossy coding (see also [5], [6], and other works) . It is demonstrated that the residual redundancy in the source (in the form of non-uniform distribution and/or memory) and the channel noise can be used to improve performance in terms of the signal-to-distortion ratio (SDR). It is also observed in [4] that using the channel's soft-decision information can result in significant SDR gain over hard-decision decoding, which is in line with other works showing that soft-decision decoding can increase channel capacity and system performance (e.g., see [7]-[10]).

In this paper, we extend the results of [4], where a singleuser system was considered, and study the JSCC problem of

\footnotetext{
S. P. Beheshti is with the Department of Electrical and Computer Engineering, Queens University,Kingston, ON, K7L 3N6, Canada

F. Alajaji and T. Linder are with the Department of Mathematics and Statistics and the Department of Electrical and Computer Engineering, Queens University, Kingston, ON, K7L 3N6, Canada

This work was supported in part by NSERC of Canada.
}

sending two correlated Gaussian sources over an orthogonal multiple access channel (MAC). A practical situation where two sensors separately measure a pair of correlated parameters, such as temperature and humidity, and sending them to a fusion center is one motivation for considering such a problem. Our MAC channel is defined by two orthogonal sub-channels. Each sub-channel is a point-to-point correlated Rayleigh discrete fading channel (DFC) used with antipodal signaling and softdecision (non-binary) output quantization. However, as the Rayleigh DFC is hard to treat analytically [4], we will instead use the recently introduced non-binary noise discrete channel with queue based noise (NBNDC-QB) which has been shown to efficiently model such DFC [11], [12].

We design a joint sequence MAP decoder (which is optimal in terms of sequence error probability) and implement it using a modified version of the Viterbi algorithm. Numerical results confirm that our joint MAP decoder takes advantage of the statistics of the correlated sources in addition to the channel's soft-decision information and statistical memory to accomplish better SDR. Our main theoretical contribution is an easy-tocheck analytical condition in terms of the sources and channel parameters, under which the costly delay-prone joint MAP decoder can be replaced by a straightforward instantaneous decoder with the same performance.

The rest of this paper is organized as follows. In Section II, we describe our MAC channel model by introducing the Rayleigh DFC and the NBNDC-QB models. The coding and decoding schemes are studied in Section III and a result which simplifies the joint MAP decoder to an instantaneous symbol-by-symbol decoder is established for the special case of correlated binary sources. Numerical results and discussions are presented in Section IV and the paper is concluded in Section V.

\section{Channel Model}

Here, we introduce our orthogonal MAC channel by briefly reviewing two point-to-point channel models studied in [12]: the NBNDC-QB and the Rayleigh DFC. It is shown in [11] that the general NBNDC-QB model can effectively represent the statistical behavior of the single-user Rayleigh DFC.

\section{A. Point-to-Point Rayleigh DFC.}

The single-user Rayleigh DFC is a binary-input and $2^{q}$ ary output channel defined as follows. First, a binary phaseshift keying (BPSK) modulator takes the DFC's binary input process $\left\{X_{k}\right\}_{k=1}^{\infty}, X_{k} \in \mathcal{X}=\{0,1\}$, and generates $S_{k}=$ $2 X_{k}-1 \in\{-1,1\}$ for $k=1,2, \ldots$ Then, the modulated signal is transmitted over a time-correlated flat Rayleigh fading channel with additive white Gaussian noise which produces the output $R_{k}=\sqrt{E_{s}} A_{k} S_{k}+N_{k}, k=1,2, \ldots$, where $E_{s}$ is the 
energy of signal sent over the channel, and $\left\{N_{k}\right\}$ is a sequence of independent and identically distributed (i.i.d.) Gaussian random variables of variance $N_{0} / 2$. Furthermore, $\left\{A_{k}\right\}$ is the channel's Rayleigh fading process (assumed to be independent of $\left\{N_{k}\right\}$ and the input process) with $A_{k}=\left|G_{k}\right|$, where $\left\{G_{k}\right\}$ is a time-correlated complex wide-sense stationary Gaussian process with Clarkes autocorrelation function given as a Bessel function of the normalized maximum Doppler frequency $f_{D} T$ [12], [13]. The fading channel's signal-to-noise ratio (SNR) is given by $\mathrm{SNR}=E_{s} / N_{0}$. Finally, a soft-decision demodulator processes the output $R_{k}$ and produces the DFC's channel output $Y_{k} \in \mathcal{Y}=\left\{0,1, \ldots 2^{q}-1\right\}$ using a $q$-bit uniform quantizer with step size $\Delta$ defined as $Y_{k}=j$, if $R_{k} \in\left(T_{j-1}^{\prime}, T_{j}^{\prime}\right.$, where $T_{-1}^{\prime}=-\infty, T_{j}^{\prime}=\left(j+1-2^{q-1}\right) \Delta$ for $j=0,1, \ldots, 2^{q}-2$, and $T_{2^{q}-1}^{\prime}=\infty$.

For the DFC, the conditional probabilities $q_{i, j}\left(a_{k}\right) \triangleq$ $\operatorname{Pr}\left(Y_{k}=j \mid X_{k}=i, A_{k}=a_{k}\right)$ and the $n$-fold transition probabilities $P_{D F C}^{(n)}\left(y_{1}^{n} \mid x_{1}^{n}\right) \triangleq \operatorname{Pr}\left\{Y_{1}^{n}=y_{1}^{n} \mid X_{1}^{n}=x_{1}^{n}\right\}$ can be calculated via $\left[12\right.$, eqs. (1), (2)] where $y_{1}^{n}=\left(y_{1}, y_{2}, \ldots, y_{n}\right)$ and $x_{1}^{n}=\left(x_{1}, x_{2}, \ldots, x_{n}\right)$. Unfortunately, $P_{D F C}^{(n)}\left(y_{1}^{n} \mid x_{1}^{n}\right)$ can be expressed in closed form only for $n \leq 3$; otherwise it must be found numerically. Therefore, the NBNDC-QB is introduced as a more tractable model for the DFC.

\section{B. NBNDC with queue-based noise}

The NBNDC-QB is a binary-input and $2^{q}$-ary-output channel model, where $q \geq 1$, which is described by

$$
Y_{j}=\left(2^{q}-1\right) X_{j}+(-1)^{X_{j}} Z_{j}, \quad j=1,2, \ldots,
$$

where $X_{j} \in\{0,1\}$ is the input data bit, $Y_{j} \in \mathcal{Y}=$ $\left\{0,1, \ldots 2^{q}-1\right\}$ is the channel output, and $Z_{j} \in \mathcal{Y}$ is the corresponding noise symbol which is assumed to be independent of the input. $\left\{Z_{j}\right\}$ is a generalization of the queue-based (QB) noise introduced in [14]; it is a $2^{q}$-ary stationary and ergodic $M^{t h}$-order Markov process which can be described using only $2^{q}+2$ independent parameters (typically, $q=2$ or 3 for most systems): the memory order $M$, the marginal probability distribution $\left(\rho_{0}, \rho_{1}, \ldots, \rho_{2^{q}-1}\right)$, and correlation parameters $0 \leq \epsilon<1$ and $\alpha \geq 0$. More details on the noise model can be found in [14] and [12]. The state process $\left\{S_{j}\right\}$ of the QB noise, defined by $S_{j}=\left(Z_{j}, Z_{j-1}, \ldots, Z_{j-M+1}\right)$ for $j \geq M$, is a homogeneous first-order Markov process taking values in $\left\{0,1, \ldots, 2^{q}-1\right\}^{M}$. The noise state transition probability is defined as $Q\left(s_{j} \mid s_{j-1}\right) \triangleq \operatorname{Pr}\left\{S_{j}=s_{j} \mid S_{j-1}=s_{j-1}\right\}$, where $s_{j}=$ $\left(z_{j}, z_{j-1}, \ldots, z_{j-M+1}\right)$ and $s_{j-1}=\left(z_{j}^{\prime}, z_{j-1}^{\prime}, \ldots, z_{j-M+1}^{\prime}\right)$. It is shown in [12] that for $j \geq M+1$,

$$
\begin{aligned}
Q\left(s_{j} \mid s_{j-1}\right)= & \left(\sum_{\ell=1}^{M-1} \delta_{z_{j}, z_{j-\ell}}+\alpha \delta_{z_{j}, z_{j-M}}\right) \frac{\epsilon}{M-1+\alpha} \\
& +(1-\epsilon) \rho_{z_{j}},
\end{aligned}
$$

if $z_{k-1}=z_{k}^{\prime}$ for $k=j, \ldots j-M+2$, and $Q\left(s_{j} \mid s_{j-1}\right)=0$ otherwise, where $\delta_{i, i^{\prime}}=1$ if $i=i^{\prime}$ and $\delta_{i, i^{\prime}}=0$ if $i \neq i^{\prime}$, and $\sum_{\ell=1}^{0} \triangleq 0$. The $n$-fold channel transition probabilities are $\operatorname{Pr}\left\{Z_{1}^{n}=z_{1}^{n}\right\}=\operatorname{Pr}\left\{Y_{1}^{n}=y_{1}^{n} \mid X_{1}^{n}=x_{1}^{n}\right\} \triangleq P_{Q B}^{(n)}\left(z_{1}^{n}\right)$, as given by [12, eqs. (20), (21)], where $y_{1}^{n}$ is the output sequence, $x_{1}^{n}$ the input sequence, and $z_{1}^{n}=\left(z_{1}, \ldots, z_{n}\right)$ is the sequence of corresponding noise symbols related to $x_{1}^{n}$ and $y_{1}^{n}$ according to (1). For $n=1, P_{Q B}^{(1)}\left(z_{1}\right)=\rho_{z_{1}}$ for all $z_{1} \in \mathcal{Y}$.

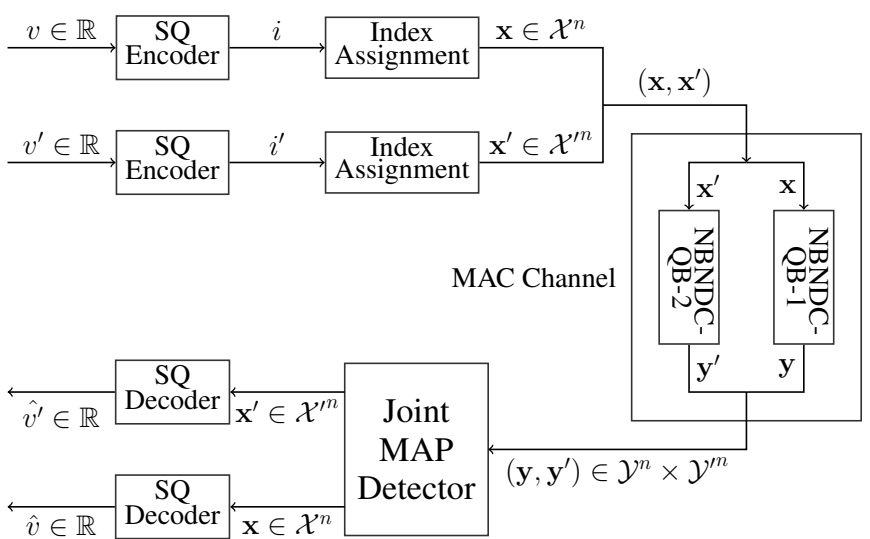

Fig. 1: Block diagram of a JSCC system using scalar quantization and joint MAP decoder over our orthogonal MAP channel with memory

The NBNDC-QB model can be fitted, via steps explained in [12], to mimic the statistical behavior of a given Rayleigh DFC with fixed parameters $\left(\mathrm{SNR}, q, \delta, f_{D} T\right)$. The memory and correlation parameters $M, \epsilon$, and $\alpha$ are coupled with $f_{D} T$; while $q, \delta$, and SNR determine the noise one-dimensional probability distributions $\rho_{j}, \forall j \in \mathcal{Y}$ as given in [4, Table I]. Consequently, both models will have the same channel noise correlation coefficient Cor, given in [12, eq. (22)].

\section{Orthogonal MAC Channel}

In many practical communication systems where the available channel bandwidth must be efficiently shared among several users, various orthogonal multiple access schemes such as frequency division multiple access, time division multiple access, and code division multiple access are employed to avoid unrecoverable collision of messages from different users. This motivates us to consider an orthogonal MAC channel consisting of two independent single-user Rayleigh DFC subchannels which are modeled via NBNDC-QB channels.

\section{MAP DETECTION OF TWO CORRELATED GAUSSIAN SOURCES OVER THE ORTHOGONAL NBNDC-QB MAC}

\section{A. System model}

Consider the communication system depicted in Fig. 1; two correlated zero-mean and unit-variance Gaussian sources $\mathcal{V}$ and $\mathcal{V}^{\prime}$ generate a sequence of input pairs $\left\{\left(V_{i}, V^{\prime}{ }_{i}\right)\right\}_{i=1}^{\infty}$ which are i.i.d. real-valued samples taken according to the bivariate normal density

$$
f_{V, V^{\prime}}\left(v, v^{\prime}\right)=\frac{1}{2 \pi \sqrt{1-\rho^{2}}} \exp \left(-\frac{v^{2}+v^{\prime 2}-2 \rho v v^{\prime}}{2\left(1-\rho^{2}\right)}\right),
$$

where $-1 \leq \rho \leq 1$ is the correlation between the two sources.

The system description in this paper is a generalization of the single-user system presented in [4]. The output samples of the first source are encoded using a rate- $n$ scalar quantizer (SQ). The SQ utilizes the Lloyd-Max algorithm [15], with the initial codebook selection obtained via the splitting algorithm [16] and produces an index $i \in\left\{0,1, \ldots, 2^{n}-1\right\}$. As explained in [4], because of its simplicity and good performance, the folded binary code (FBC) [17] is chosen as the one-to-one index assignment method to map the index $i$ to a binary vector $\mathbf{x} \in\{0,1\}^{n}$. The same encoding process is separately done for the second source which results in the codeword 
$\mathbf{x}^{\prime} \in\{0,1\}^{n}$. Then, the vector pair $\left(\mathbf{x}, \mathbf{x}^{\prime}\right)$ is transmitted through the orthogonal Rayleigh DFC MAC channel and the corresponding vectors $\mathbf{y} \in \mathcal{Y}^{n}=\left\{0,1, \ldots, 2^{q}-1\right\}^{n}$ and $\mathbf{y}^{\prime} \in \mathcal{Y}^{\prime n}=\left\{0,1, \ldots, 2^{q^{\prime}}-1\right\}^{n}$ are received. This communication is modeled as sending the $n$-tuple codeword $\mathbf{x}$ bit-by-bit over the first NBNDC-QB sub-channel with $2^{q}$ ary noise symbols $z \in \mathcal{Y}=\left\{0,1, \ldots, 2^{q}-1\right\}$ and noise memory $M$ which will result in the output sequence $\mathbf{y}$. Similarly, $\mathbf{x}^{\prime}$ and $\mathbf{y}^{\prime}$ are the input and output vectors of the second NBNDC-QB sub-channel with $2^{q^{\prime}}$-ary noise symbols $z^{\prime} \in \mathcal{Y}^{\prime}=\left\{0,1, \ldots, 2^{q^{\prime}}-1\right\}$ and noise memory $M^{\prime}$. At the receiver side, the MAC channel's output $\left(\mathbf{y}, \mathbf{y}^{\prime}\right)$ is fed to a joint MAP decoder. Finally, two SQ decoders map the decoder outputs $\left(\hat{\mathbf{x}}, \hat{\mathbf{x}}^{\prime}\right)$ into output levels of the quantizer codebook.

It can be observed that in the described system, which is referred to as the SQ-MAC-MAP system, the receiver carries most of the complexity load.

\section{B. MAP decoder design}

The residual redundancy of the source and channel statistics can be harnessed by a MAP decoder which is designed to minimize the sequence error probability [17]. Suppose that each source produces $N$ symbols. The sequence $\left(\mathbf{x}, \mathbf{x}^{\prime}\right)^{N}=\left(\left(\mathbf{x}_{1}, \mathbf{x}^{\prime}{ }_{1}\right), \ldots,\left(\mathbf{x}_{N}, \mathbf{x}^{\prime}{ }_{N}\right)\right) \in(\{0,1\} \times\{0,1\})^{n N}$ at the output of the SQ encoder is transmitted over the MAC channel in $n N$ channel uses. The independent NBNDC-QB sub-channels contaminate the bit streams related to the first and second source with noise sequences $z_{1}^{n N} \in \mathcal{Y}^{n N}$ and ${z^{\prime}}_{1}^{n N} \in \mathcal{Y}^{\prime n N}$, respectively. Receiving the channel output $\left(\mathbf{y}, \mathbf{y}^{\prime}\right)^{N}=\left(\left(\mathbf{y}_{1}, \mathbf{y}^{\prime}{ }_{1}\right), \ldots,\left(\mathbf{y}_{N}, \mathbf{y}^{\prime}{ }_{N}\right)\right) \in\left(\mathcal{Y} \times \mathcal{Y}^{\prime}\right)^{n N}$, the MAP decoder estimates $\left(\mathbf{x}, \mathbf{x}^{\prime}\right)^{N}$ by $\left(\hat{\mathbf{x}}, \hat{\mathbf{x}}^{\prime}\right)^{N}$ as

$$
\begin{aligned}
& \left(\hat{\mathbf{x}}, \hat{\mathbf{x}}^{\prime}\right)^{N}= \\
& \underset{\left(\mathbf{x}, \mathbf{x}^{\prime}\right)^{N}}{\arg \max }\left(\operatorname{Pr}\left\{\left(\mathbf{X}, \mathbf{X}^{\prime}\right)^{N}=\left(\mathbf{x}, \mathbf{x}^{\prime}\right)^{N} \mid\left(\mathbf{Y}, \mathbf{Y}^{\prime}\right)^{N}=\left(\mathbf{y}, \mathbf{y}^{\prime}\right)^{N}\right\}\right) \\
& =\underset{\left(\mathbf{x}, \mathbf{x}^{\prime}\right)^{N}}{\arg \max }\left(\operatorname{Pr}\left\{\left(\mathbf{Y}, \mathbf{Y}^{\prime}\right)^{N}=\left(\mathbf{y}, \mathbf{y}^{\prime}\right)^{N} \mid\left(\mathbf{X}, \mathbf{X}^{\prime}\right)^{N}=\left(\mathbf{x}, \mathbf{x}^{\prime}\right)^{N}\right\}\right. \\
& \left(\mathbf{x}, \mathbf{x}^{\prime}\right)^{N} \\
& \left.\times \operatorname{Pr}\left\{\left(\mathbf{X}, \mathbf{X}^{\prime}\right)^{N}=\left(\mathbf{x}, \mathbf{x}^{\prime}\right)^{N}\right\}\right) \\
& =\underset{\left(\mathbf{x}, \mathbf{x}^{\prime}\right)^{N}}{\arg \max }\left(\operatorname{Pr}\left\{\mathbf{Y}^{N}=\mathbf{y}^{N} \mid \mathbf{X}^{N}=\mathbf{x}^{N}\right\} \times\right. \\
& \left.\operatorname{Pr}\left\{\mathbf{Y}^{\prime N}=\mathbf{y}^{\prime N} \mid \mathbf{X}^{\prime N}=\mathbf{x}^{\prime N}\right\} \operatorname{Pr}\left\{\left(\mathbf{X}, \mathbf{X}^{\prime}\right)^{N}=\left(\mathbf{x}, \mathbf{x}^{\prime}\right)^{N}\right\}\right) \\
& =\arg \max \left(\operatorname{Pr}\left\{Z_{1}^{n N}=z_{1}^{n N}\right\} \operatorname{Pr}\left\{Z_{1}^{\prime n N}=z_{1}^{\prime n N}\right\} \times\right. \\
& \left(\mathbf{x}, \mathbf{x}^{\prime}\right)^{N} \\
& \left.\operatorname{Pr}\left\{\left(\mathbf{X}, \mathbf{X}^{\prime}\right)^{N}=\left(\mathbf{x}, \mathbf{x}^{\prime}\right)^{N}\right\}\right) \\
& =\underset{\left(\mathbf{x}, \mathbf{x}^{\prime}\right)^{N}}{\arg \max }\left(P_{Q B}^{(n)}\left(z_{1}^{n}\right) P_{Q B}^{\prime(n)}\left(z_{1}^{\prime n}\right) P\left(\mathbf{x}_{1}, \mathbf{x}^{\prime}{ }_{1}\right) \times\right. \\
& \left.\prod_{i=1}^{N-1}\left(Q\left(z_{i n+1}^{(i+1) n} \mid z_{1}^{i n}\right) Q^{\prime}\left(z_{i n+1}^{\prime(i+1) n} \mid z_{1}^{\prime i n}\right) P\left(\mathbf{x}_{i+1}, \mathbf{x}_{i+1}^{\prime}\right)\right)\right),
\end{aligned}
$$

where the third equation comes from the orthogonality of the two sub-channels. $P\left(\mathbf{x}_{i+1}, \mathbf{x}_{i+1}^{\prime}\right) \triangleq P\left(\mathbf{X}_{i+1}=\right.$ $\left.\mathbf{x}_{i+1}, \mathbf{X}_{i+1}^{\prime}=\mathbf{x}_{i+1}^{\prime}\right)$ is the joint probability distribution for the pair of $n$-tuple codewords $\left(\mathbf{X}_{i+1}, \mathbf{X}_{i+1}^{\prime}\right)$. For $i=$ $1,2, \ldots, n N$, the noise symbols $z_{i}$ and $z^{\prime}{ }_{i}$ can be found using (1) with respect to each sub-channel inputs and outputs. The transition probabilities in the last line of (4) are defined based on $Q\left(z_{i+1}^{i+j} \mid z_{i-k}^{i}\right) \triangleq \operatorname{Pr}\left\{Z_{i+1}^{i+j}=z_{i+1}^{i+j} \mid Z_{i-k}^{i}=z_{i-k}^{i}\right\}$, where $i, j, k \in\{1,2, \ldots, n N-1\}, i+j \leq n N, i-k \geq 1$. Note that $z_{i} \triangleq 0$ if $i<1, z_{i}^{j} \triangleq\left(z_{i}, z_{i+1}, \ldots, z_{j}\right), j \geq i$. For the second sub-channel, $Q\left({z^{\prime}}_{i+1}^{i+j} \mid z^{\prime i-k}\right)$ and $P_{Q B}^{\prime(n)}\left({z^{\prime}}_{1}^{n}\right)$ are defined and calculated similarly using the parameters associated with this channel.

Assuming $n N \geq \max \left\{M, M^{\prime}\right\}$, it can be shown that

$$
\begin{aligned}
\left(\mathbf{x}, \mathbf{x}^{\prime}\right)^{N}= & \underset{\left(\mathbf{x}, \mathbf{x}^{\prime}\right)^{N}}{\arg \max }\left\{\log \left[P_{Q B}^{(n)}\left(z_{1}^{n}\right) P_{Q B}^{\prime(n)}\left(z_{1}^{\prime n}\right) P\left(\mathbf{x}_{1}, \mathbf{x}^{\prime}{ }_{1}\right)\right]\right. \\
& +\sum_{i=1}^{N-1} \log \left[Q\left(z_{i n+1}^{(i+1) n} \mid z_{i n-(M-1)}^{i n}\right) \times\right. \\
& \left.\left.Q^{\prime}\left(z^{\prime(i n+1) n} \mid z_{i n-\left(M^{\prime}-1\right)}^{\prime i n}\right) P\left(\mathbf{x}_{i+1}, \mathbf{x}^{\prime}{ }_{i+1}\right)\right]\right\}
\end{aligned}
$$

where $Q\left(z_{i n+1}^{(i+1) n} \mid z_{i n-(M-1)}^{i n}\right)$ can be calculated via [4, eq. (9)].

To implement the MAP decoder, we employ a modified version of the Viterbi algorithm similar to the one used in [18]. The corresponding trellis consists of $4^{(k n)}$ states, where $k$ is the smallest integer which satisfies $k n \geq \max \left\{M, M^{\prime}\right\}$, each with the following path metric at step $i$ :

$$
\begin{aligned}
& \log \left[Q\left(z_{i n+1}^{(i+1) n} \mid z_{i n-(M-1)}^{i n}\right) Q^{\prime}\left(z_{i n+1}^{\prime(i+1) n} \mid z_{i n-\left(M^{\prime}-1\right)}^{\prime i n}\right)\right] \\
& +\log \left[P\left(\mathbf{x}_{i+1}, \mathbf{x}_{i+1}^{\prime}\right)\right] .
\end{aligned}
$$

\section{Case study: MAP detection of binary sources}

Applying the Viterbi algorithm, the MAP decoder needs to observe the entire received sequence before deciding on the most likely message words, which results in significant decoding delay as well as storage complexity that increases with the length of the sequence. Thus it is interesting to investigate situations where MAP decoding can be replaced by a simple and fast instantaneous (symbol-by-symbol) decoding rule which exhibits the same performance in terms of symbol error rate (SER). For the single-user case and for a binary symmetric Markov source transmitted over a NBNDC-QB channel with noise memory $M=1$, [4] establishes a necessary and sufficient condition under which an instantaneous symbolby-symbol decoder can function as the MAP decoder.

In our MAC problem, we consider the special case where both correlated sources are separately quantized to two levels $(n=1)$. Using the symmetry in the joint distribution (3), one can verify the following equations for the joint distribution of the resulting binary sources:

$$
P(1,1)=P(0,0) \quad P(1,0)=P(0,1)
$$

In the next step, we explain our instantaneous symbol-bysymbol decoder. By making use of the orthogonality of the MAC channel, equation (6), and the assumption $\rho_{0} \geq \rho_{1} \geq$ $\rho_{2} \geq \cdots \geq \rho_{2^{q}-1}$ for each NBNDC-QB sub-channel, it can be shown that for the case of binary sources, the same function $\theta$ introduced in [4] can be adapted to map a $2^{q}$-ary $(q \geq 1)$ output $y_{i}$ of the NBNDC-QB channel to a binary symbol $\tilde{y}_{i}$. In fact, among all mappings $\theta: \mathcal{Y} \mapsto\{0,1\}$, the following mapping $\theta^{*}$ minimizes the symbol error probability for each sub-channel:

$$
\theta^{*}\left(y_{i}\right)=\tilde{y}_{i}=\left\{\begin{array}{ll}
0, & \text { if } y_{i}<2^{q-1} \\
1, & \text { otherwise }
\end{array} \quad ; 0 \leq i \leq N\right.
$$

Note that we independently apply the same function (7) 
to $y$ and $y^{\prime}$, the demodulated outputs of the orthogonal MAC. Since the parameters of the NBNDC-QB sub-channels can be different, we denote the first instantaneous decoder by $\theta^{*}$ and the second by $\theta^{\prime *}$ with the $q$ in (7) changed to $q^{\prime}$. Hence, a joint symbol $\left(y_{i}, y_{i}^{\prime}\right)$ is decoded correctly when $\left(\tilde{y}_{i}, \tilde{y}_{i}^{\prime}\right)=\left(x_{i}, x_{i}^{\prime}\right)$.

The following result presents a necessary and sufficient condition for the mappings $\left(\theta^{*}, \theta^{\prime *}\right)$ to form an optimal sequence detection rule in the sense of minimizing the sequence error probability.

Theorem 1. Consider two correlated memoryless binary sources having joint distribution $P\left(x, x^{\prime}\right)$ with the symmetry assumption (6) and an orthogonal MAC channel consisting of two independent NBNDC-QB sub-channels where the first one has the correlation parameter $\epsilon \geq 0$, memory order $M=1$, $q \geq 1$, and a noise one-dimensional probability distribution satisfying $\rho_{0} \geq \rho_{1} \geq \rho_{2} \geq \cdots \geq \rho_{2^{q}-1}$. Similarly, assume that in the second channel $\epsilon^{\prime} \geq 0, q^{\prime} \geq 1, M^{\prime}=1$, and $\rho_{0}^{\prime} \geq \rho_{1}^{\prime} \geq \rho_{2}^{\prime} \geq \cdots \geq \rho_{2^{q^{\prime}-1}}^{\prime}$. Let $\left(\bar{x}, x^{\prime}\right)^{N}$ be a source sequence of length $N \geq 2,\left(y, y^{\prime}\right)^{N}$ the channel output sequence, and let $\left(\tilde{y}, \tilde{y}^{\prime}\right)^{\bar{N}}=\left(\theta^{*}(y), \theta^{\prime *}\left(y^{\prime}\right)\right)^{N}$ be obtained by applying the mapping functions component-wise to the corresponding output sequences of the underlying channels.

Then, decoding as $\left(\hat{x}, \hat{x}^{\prime}\right)^{N}=\left(\tilde{y}, \tilde{y}^{\prime}\right)^{N}$ is an optimal sequence MAP detection rule for all possible received sequences if

$$
\min \left\{\left(\frac{P(0,0)}{\frac{1}{2}-P(0,0)}\right),\left(\frac{\frac{1}{2}-P(0,0)}{P(0,0)}\right)\right\} A \geq 1,
$$

where

$A=\min \left\{\frac{\epsilon^{\prime}+\left(1-\epsilon^{\prime}\right) \rho_{2^{q^{\prime}-1}-1}^{\prime}}{\epsilon^{\prime}+\left(1-\epsilon^{\prime}\right) \rho_{2^{q^{\prime}-1}}^{\prime}}, \frac{\epsilon+(1-\epsilon) \rho_{2^{q-1}-1}}{\epsilon+(1-\epsilon) \rho_{2^{q-1}}}\right\}$.

Conversely, if (8) does not hold, it can be shown that there is at least one sequence for which $\left(\hat{x}, \hat{x}^{\prime}\right)^{N}=\left(\tilde{y}, \tilde{y}^{\prime}\right)^{N}$ is not an optimal sequence MAP detection rule.

\section{NumERicAl Results}

In order to illustrate Theorem 1, we have simulated the system by generating $N=10^{5}$ independent samples of two correlated binary sources according to a joint distribution satisfying (6). Denoting the left-hand term of (8) by $C$, when $C \geq 1$ it can be observed from Table I that the performance of the instantaneous decoding $\left(\theta^{*}, \theta^{* *}\right)$ and the joint MAP decoder are identical, while for $C<1$ the joint MAP decoder outperforms the instantaneous decoder. In Table I. (a), we present results for the cases when the MAC's sub-channels have identical parameters and in Table I. (b) we use subchannels with different parameters in order to further illustrate the theoretical results.

We also simulated the SQ-MAC-MAP system for sending two correlated Gaussian sources (generated by (3)). Table II shows the average SDR (in $\mathrm{dB}$ ) in the system simulation, where SDR is defined as

$$
S D R \triangleq \frac{\sum_{i=1}^{2} E\left[\left(\mathbf{X}_{i}\right)^{2}\right]}{\sum_{i=1}^{2} E\left[\left(\mathbf{X}_{i}-\hat{\mathbf{X}}_{i}\right)^{2}\right]}
$$

It can be verified that in general the system performs better with highly correlated sources and high noise correlation which means that the joint MAP decoder successfully exploits the channel noise correlation and the correlation between sources. For example, when the correlation between sources is high (0.81), a $5.4 \mathrm{~dB}$ (at $q=2, n=3, \mathrm{SNR}=2$ ) SDR gain is achieved by having a system with high noise correlation Cor $=0.9$ (instead of a fully-interleaved memoryless channel); also, in such a system, increasing the correlation between sources (from -0.31 to 0.81 ) leads to significant improvements as high as $4.2 \mathrm{~dB}$ (at $q=2, n=3, \mathrm{SNR}=2$ ) in SDR.

Furthermore, it can be observed that incorporating more soft-decision information has a positive effect on the performance of the system under joint MAP decoding. For example using a 3-bit soft-decision quantizer rather a hard-decision quantizer $(q=1)$ results in a $4.28 \mathrm{~dB}$ gain (at $n=3, \mathrm{SNR}=2$, Cor $=0.9$ and $\rho=0.81)$.

Considering Table III, increasing soft-decision information and also the correlation between the sources do not have any significant effect on the performance of the instantaneous decoder. These results are predictable because according to (7), for $0 \leq i \leq N$, the outputs of the instantaneous symbol-bysymbol decoder $\left(\theta^{*}\left(y_{i}\right), \theta^{\prime *}\left(y_{i}^{\prime}\right)\right)$ can be written as functions of $R_{i}$ and $R_{i}^{\prime}$, the unquantized outputs of the Rayleigh fading underlying sub-channels

$$
\tilde{y}_{i}=\left\{\begin{array}{ll}
0, & \text { if } R_{i} \leq 0 \\
1, & \text { otherwise }
\end{array}, \quad \tilde{y}_{i}^{\prime}= \begin{cases}0, & \text { if } R_{i}^{\prime} \leq 0 \\
1, & \text { otherwise }\end{cases}\right.
$$

which shows no dependence on the $q, q^{\prime}$, and $P\left(x, x^{\prime}\right)$.

Furthermore, considering a system with a 2-level quantizer $(n=1)$ and a fully interleaved channel $(C o r=0)$, we can verify Theorem 1 by comparing Tables II and III and observing that whenever $C \geq 1$ in Table I, the instantaneous symbolby-symbol decoder is performing as well as the joint MAP decoder.

\section{CONCLUSION}

In this paper, we studied the joint MAP decoding for a system with two correlated Gaussian sources, scalar quantizers, and an orthogonal Rayleigh DFC MAC modeled with two independent NBNDC-QB sub-channels. Considering two-level quantizers, we derived a necessary and sufficient condition under which our instantaneous symbol-by-symbol decoder can replace the joint MAP decoder without loss of optimality. Finally, numerical results illustrate our theoretical result and verify that the proposed system can make use of the sources' correlation, channel noise memory, and channel soft-decision information to improve SDR performance. In future work, we plan to extend the theorem for a similar system with Markovian sources. Furthermore, investigating the system when the channel's memory order is greater than one as well as evaluating the system's effectiveness under MAP decoding by fitting it to the underlying fading channel are interesting research directions.

\section{REFERENCES}

[1] C. E. Shannon, "Coding theorems for a discrete source with a fidelity criterion," IRE Nat. Conv. Rec, vol. 4, pp. 142-163, 1959.

[2] Y. Zhong, F. Alajaji, and L. Campbell, "On the joint source-channel coding error exponent for discrete memoryless systems," IEEE Trans. Inf. Theory, vol. 52, no. 4, pp. 1450-1468, 2006.

[3] J. Lim and D. Neuhoff, "Joint and tandem source-channel coding with complexity and delay constraints," IEEE Trans. Commun., vol. 51, no. 5, pp. 757-766, 2003.

[4] S. Shahidi, F. Alajaji, and T. Linder, "MAP detection and robust lossy coding over soft-decision correlated fading channels," IEEE Trans. Veh. Technol., vol. 62, no. 7, pp. 3175-3187, 2013. 
TABLE I: JOINT SYMBOL ERROR RATE (IN \%) OF JOINT MAP DECODING AND INSTANTANEOUS MAPPING $\left(\theta^{*}, \theta^{\prime *}\right)$ FOR TWO CORRELATED SOURCES WITH THE JOINT DISTRIBUTIONS $P(0,0)=$ 0.2 AND 0.4. THE CHANNEL MODEL IS A MAC CHANNEL WITH TWO ORTHOGONAL NBNDC-QB, WITH $M=1$, Cor $=0.0$, AND $q=1,2,3$.

PART (a): TWO SUB-CHANNELS WITH IDENTICAL PARAMETERS (SNR, $q$ ).

\begin{tabular}{|c|c|c|c|c|c|c|c|c|c|}
\hline \multirow{3}{*}{$P(0,0)$} & \multirow{3}{*}{$q$} & \multicolumn{8}{|c|}{ SNR (dB) } \\
\hline & & \multicolumn{2}{|r|}{15} & \multicolumn{2}{|c|}{10} & \multicolumn{2}{|c|}{5} & \multicolumn{2}{|r|}{2} \\
\hline & & MAP & $\left(\theta^{*}, \theta^{\prime *}\right)$ & MAP & $\left(\theta^{*}, \theta^{\prime *}\right)$ & MAP & $\left(\theta^{*}, \theta^{\prime *}\right)$ & MAP & $\left(\theta^{*}, \theta^{\prime *}\right)$ \\
\hline \multirow{6}{*}{0.2} & \multirow{2}{*}{1} & \multicolumn{2}{|c|}{$C=85.66>1$} & \multicolumn{2}{|c|}{$C=27.98>1$} & \multicolumn{2}{|c|}{$C=9.72>1$} & \multicolumn{2}{|c|}{$C=5.48>1$} \\
\hline & & 1.51 & 1.51 & 4.63 & 4.63 & 12.44 & 12.44 & 20.52 & 20.52 \\
\hline & \multirow{2}{*}{2} & \multicolumn{2}{|c|}{$C=2.08>1$} & \multicolumn{2}{|c|}{$C=1.88>1$} & \multicolumn{2}{|c|}{$C=1.90>1$} & \multicolumn{2}{|c|}{$C=1.54>1$} \\
\hline & & 1.53 & 1.53 & 4.60 & 4.60 & 12.44 & 12.44 & 20.52 & 20.52 \\
\hline & \multirow{2}{*}{3} & \multicolumn{2}{|c|}{$C=1.20>1$} & \multicolumn{2}{|c|}{$C=1.19>1$} & \multicolumn{2}{|c|}{$C=1.09>1$} & \multicolumn{2}{|c|}{$C=1.03>1$} \\
\hline & & 1.53 & 1.53 & 4.61 & 4.61 & 12.48 & 12.48 & 20.54 & 20.54 \\
\hline \multirow{6}{*}{0.4} & \multirow{2}{*}{1} & \multicolumn{2}{|c|}{$C=32.12>1$} & \multicolumn{2}{|c|}{$C=10.49>1$} & \multicolumn{2}{|c|}{$C=3.64>1$} & \multicolumn{2}{|c|}{$C=2.05>1$} \\
\hline & & 1.53 & 1.53 & 4.61 & 4.61 & 12.48 & 12.48 & 20.56 & 20.56 \\
\hline & \multirow{2}{*}{2} & \multicolumn{2}{|c|}{$C=0.78<1$} & \multicolumn{2}{|c|}{$C=0.70<1$} & \multicolumn{2}{|c|}{$C=0.71<1$} & \multicolumn{2}{|c|}{$C=0.58<1$} \\
\hline & & 1.32 & 1.53 & 3.77 & 4.60 & 10.28 & 12.44 & 15.94 & 20.45 \\
\hline & \multirow{2}{*}{3} & \multicolumn{2}{|c|}{$C=0.45<1$} & $C=$ & $.45<1$ & $C=$ & $.41<1$ & $C=$ & $.38<1$ \\
\hline & & 1.17 & 1.54 & 3.47 & 4.58 & 9.50 & 12.43 & 15.66 & 20.48 \\
\hline
\end{tabular}

PART (b): TWO SUB-CHANNELS WITH DIFFERENT PARAMETERS (SNR, $q$ ) AND $\left(\operatorname{SNR}^{\prime}, q^{\prime}\right)$, RESPECTIVELY.

\begin{tabular}{|c|c|c|c|c|c|c|c|c|c|}
\hline \multirow{3}{*}{$P(0,0)$} & \multirow{3}{*}{$\left(q, q^{\prime}\right)$} & \multicolumn{8}{|c|}{$\left(\mathrm{SNR}, \mathrm{SNR}^{\prime}\right)(\mathrm{dB})$} \\
\hline & & \multicolumn{2}{|c|}{$(15,2)$} & \multicolumn{2}{|c|}{$(10,5)$} & \multicolumn{2}{|c|}{$(5,10)$} & \multicolumn{2}{|c|}{$(2,15)$} \\
\hline & & MAP & $\left(\theta^{*}, \theta^{\prime *}\right)$ & MAP & $\left(\theta^{*}, \theta^{\prime *}\right)$ & MAP & $\left(\theta^{*}, \theta^{\prime *}\right)$ & MAP & $\left(\theta^{*}, \theta^{\prime *}\right)$ \\
\hline \multirow{4}{*}{0.2} & \multirow{2}{*}{$(1,3)$} & \multicolumn{2}{|c|}{$C=1.03>1$} & \multicolumn{2}{|c|}{$C=1.09>1$} & \multicolumn{2}{|c|}{$C=1.19>1$} & \multicolumn{2}{|c|}{$C=1.20>1$} \\
\hline & & 11.53 & 11.53 & 8.63 & 8.63 & 8.60 & 8.60 & 11.53 & 11.53 \\
\hline & \multirow{2}{*}{$(1,2)$} & \multicolumn{2}{|c|}{$C=1.54>1$} & \multicolumn{2}{|c|}{$C=1.90>1$} & \multicolumn{2}{|c|}{$C=1.88>1$} & \multicolumn{2}{|c|}{$C=2.08>1$} \\
\hline & & 11.50 & 11.50 & 8.63 & 8.63 & 8.59 & 8.59 & 11.52 & 11.52 \\
\hline \multirow{4}{*}{0.4} & \multirow[b]{2}{*}{$(1,3)$} & \multicolumn{2}{|c|}{$C=0.38<1$} & \multicolumn{2}{|c|}{$C=0.41<1$} & \multicolumn{2}{|c|}{$C=0.45<1$} & \multicolumn{2}{|c|}{$C=0.45<1$} \\
\hline & & 8.68 & 11.51 & 7.08 & 8.60 & 6.97 & 7.15 & 11.43 & 11.60 \\
\hline & \multirow{2}{*}{$(1,2)$} & \multicolumn{2}{|c|}{$C=0.58<1$} & \multicolumn{2}{|c|}{$C=0.71<1$} & \multicolumn{2}{|c|}{$C=0.70<1$} & \multicolumn{2}{|c|}{$C=0.78<1$} \\
\hline & & 8.65 & 11.50 & 7.38 & 8.58 & 8.19 & 8.64 & 11.45 & 11.55 \\
\hline
\end{tabular}

[5] M. Skoglund, "Soft decoding for vector quantization over noisy channels with memory," IEEE Trans. Inf. Theory, vol. 45, no. 4, pp. 12931307, 1999.

[6] D. Miller and M. Park, "A sequence-based approximate MMSE decoder for source coding over noisy channels using discrete hidden Markov models," IEEE Trans. Commun., vol. 46, no. 2, pp. 222-231, 1998.

[7] N. Phamdo and F. Alajaji, "Soft-decision demodulation design for COVQ over white, colored, and ISI Gaussian channels," IEEE Trans. Commun., vol. 48, no. 9, pp. 1499-1506, 2000.

[8] F. Behnamfar, F. Alajaji, and T. Linder, "Channel-optimized quantization with soft-decision demodulation for space-time orthogonal blockcoded channels," IEEE Trans. Signal Process., vol. 54, no. 10, pp. 39353946, 2006.

[9] N. Wernersson and M. Skoglund, "On source decoding based on finitebandwidth soft information," in Proc. IEEE Int. Symp. Inf. Theory, 2005, pp. 87-91.

[10] J. Singh, O. Dabeer, and U. Madhow, "On the limits of communication with low-precision analog-to-digital conversion at the receiver," IEEE Trans. Commun., vol. 57, no. 12, pp. 3629-3639, 2009.

[11] C. Pimentel, F. Alajaji, and P. Melo, "A discrete queue-based model for soft-decision demodulated correlated fading channels," in Vehicular Technology Conference (VTC Spring), 2011 IEEE 73rd, 2011, pp. 1-5.

[12] _ " "A discrete queue-based model for capturing memory and softdecision information in correlated fading channels," IEEE Trans. Commun., vol. 60, no. 6, pp. 1702-1711, 2012.

[13] R. H. Clarke, "A statistical theory of mobile radio reception," Bell Systems Technical Journal, vol. 47, no. 6, pp. 957-1000, 1968.

[14] Z. Libo, F. Alajaji, and G. Takahara, "A binary communication channel with memory based on a finite queue," IEEE Trans. Inf. Theory, vol. 53, no. 8, pp. 2815-2840, 2007.

[15] S. Lloyd, "Least squares quantization in PCM," IEEE Trans. Inf. Theory, vol. 28, no. 2, pp. 129-137, 1982.
TABLE II: SQ-MAC-MAP TRAINING SDR RESULTS (IN DB) FOR TWO CORRELATED SOURCES WITH CORRELATION PARAMETERS $\rho=-0.31$ and $\rho=0.81$ AND CORRESPONDING JOINT DISTRIBUTIONS $P(0,0)=0.2$ AND $P(0,0)=0.4$ SENT OVER THE ORTHOGONAL MAC CHANNEL WITH MEMORYLESS NBNDC-QB SUBCHANNELS $(C o r=0.0)$ AND HIGHLY CORRELATED NBNDC-QB SUB-CHANNELS (Cor $=0.9$ ) WITH PARAMETERS $M=\alpha=1$.

\begin{tabular}{|c|c|c|c|c|c|c|c|c|c|c|}
\hline \multirow{3}{*}{$\begin{array}{c}\text { Sources } \\
\text { Correlation }\end{array}$} & \multirow{3}{*}{$q$} & \multirow{3}{*}{$n$} & \multicolumn{4}{|c|}{ Fully interleaved (Cor=0) } & \multicolumn{4}{|c|}{ Cor $=0.9$} \\
\hline & & & & SNR & & & & & & \\
\hline & & & 15 & 10 & 5 & 2 & 15 & 10 & 5 & 2 \\
\hline \multirow{9}{*}{-0.31} & \multirow{3}{*}{1} & 1 & 4.17 & 3.74 & 2.78 & 1.94 & 4.17 & 3.74 & 2.78 & 1.94 \\
\hline & & 2 & 8.15 & 6.48 & 3.83 & 2.13 & 8.32 & 6.91 & 4.65 & 3.19 \\
\hline & & 3 & 11.02 & 7.74 & 3.85 & 1.89 & 11.47 & 8.51 & 5.17 & 3.34 \\
\hline & \multirow{3}{*}{2} & 1 & 4.17 & 3.74 & 2.78 & 1.94 & 4.22 & 3.91 & 3.09 & 2.31 \\
\hline & & 2 & 8.23 & 6.60 & 3.97 & 2.45 & 8.80 & 7.87 & 6.03 & 4.56 \\
\hline & & 3 & 11.26 & 8.16 & 4.45 & 2.57 & 12.92 & 10.54 & 7.27 & 5.19 \\
\hline & \multirow{3}{*}{3} & 1 & 4.17 & 3.74 & 2.78 & 1.94 & 4.24 & 3.93 & 3.20 & 2.43 \\
\hline & & 2 & 8.23 & 6.68 & 4.15 & 2.53 & 8.89 & 8.11 & 6.42 & 5.08 \\
\hline & & 3 & 11.43 & 8.31 & 4.68 & 2.70 & 13.14 & 11.14 & 8.03 & 6.02 \\
\hline \multirow{9}{*}{0.81} & \multirow{3}{*}{1} & 1 & 4.17 & 3.74 & 2.78 & 1.94 & 4.16 & 3.72 & 2.75 & 1.90 \\
\hline & & 2 & 8.11 & 6.46 & 4.25 & 2.66 & 8.90 & 8.19 & 6.75 & 5.53 \\
\hline & & 3 & 11.01 & 7.64 & 4.01 & 2.34 & 13.22 & 11.47 & 8.44 & 6.46 \\
\hline & \multirow{3}{*}{2} & 1 & 4.28 & 4.03 & 3.33 & 2.59 & 4.34 & 4.22 & 3.90 & 3.32 \\
\hline & & 2 & 8.73 & 7.67 & 5.52 & 3.70 & 9.20 & 8.94 & 8.24 & 7.33 \\
\hline & & 3 & 12.64 & 10.02 & 6.33 & 3.99 & 14.25 & 13.41 & 11.47 & 9.42 \\
\hline & \multirow{3}{*}{3} & 1 & 4.26 & 3.98 & 3.24 & 2.65 & 4.37 & 4.31 & 4.07 & 3.72 \\
\hline & & 2 & 8.84 & 7.94 & 5.82 & 3.99 & 9.23 & 9.12 & 8.61 & 8.01 \\
\hline & & 3 & 13.00 & 10.63 & 6.87 & 4.40 & 14.40 & 13.92 & 12.44 & 10.74 \\
\hline
\end{tabular}

TABLE III: SQ WITH INSTANTANEOUS DECODER $\left(\theta^{*}, \theta^{\prime *}\right)$ - TRAINING SDR RESULTS (IN DB) FOR TWO CORRELATED SOURCES WITH CORRELATION PARAMETERS $\rho=-0.31$ and $\rho=0.81$ AND CORRESPONDING JOINT DISTRIBUTIONS $P(0,0)=0.2$ AND $P(0,0)=0.4$ SENT OVER THE ORTHOGONAL MAC CHANNEL WITH MEMORYLESS NBNDC-QB SUB-CHANNELS ( $\mathrm{Cor}=0.0)$ AND HIGHLY CORRELATED NBNDC-QB SUB-CHANNELS $(\mathrm{Cor}=0.9)$ WITH PARAMETERS $M=\alpha=1$.

\begin{tabular}{|c|c|c|c|c|c|c|c|c|c|c|}
\hline \multirow{3}{*}{$\begin{array}{c}\text { Sources } \\
\text { Correlation }\end{array}$} & \multirow{3}{*}{$q$} & \multirow{3}{*}{$n$} & \multirow{2}{*}{\multicolumn{4}{|c|}{$\frac{\text { Fully interleaved }(\text { Cor }=0)}{\text { SNR }(\mathrm{dB})}$}} & \multicolumn{4}{|c|}{ Cor $=0.9$} \\
\hline & & & & & & & & SNR & & \\
\hline & & & 15 & 10 & 5 & 2 & 15 & 10 & 5 & 2 \\
\hline \multirow{9}{*}{-0.31} & \multirow{3}{*}{1} & 1 & 4.17 & 3.74 & 2.78 & 1.94 & 4.17 & 3.74 & 2.78 & 1.94 \\
\hline & & 2 & 8.15 & 6.48 & 3.83 & 2.13 & 8.28 & 6.77 & 4.30 & 2.64 \\
\hline & & 3 & 11.02 & 7.74 & 3.96 & 1.85 & 11.25 & 8.09 & 4.41 & 2.29 \\
\hline & \multirow{3}{*}{2} & 1 & 4.17 & 3.74 & 2.78 & 1.94 & 4.17 & 3.74 & 2.80 & 1.97 \\
\hline & & 2 & 8.16 & 6.47 & 3.86 & 2.15 & 8.26 & 6.79 & 4.35 & 2.63 \\
\hline & & 3 & 11.02 & 7.75 & 3.95 & 1.84 & 11.28 & 8.06 & 4.36 & 2.29 \\
\hline & \multirow{3}{*}{3} & 1 & 4.17 & 3.74 & 2.78 & 1.94 & 4.15 & 3.72 & 2.77 & 1.96 \\
\hline & & 2 & 8.13 & 6.48 & 3.84 & 2.13 & 8.32 & 6.81 & 4.28 & 2.67 \\
\hline & & 3 & 11.05 & 7.76 & 3.95 & 1.86 & 11.28 & 8.04 & 4.41 & 2.27 \\
\hline \multirow{9}{*}{0.81} & \multirow{3}{*}{1} & 1 & 4.17 & 3.74 & 2.78 & 1.94 & 4.17 & 3.73 & 2.77 & 1.94 \\
\hline & & 2 & 8.14 & 6.47 & 3.84 & 2.14 & 8.31 & 6.74 & 4.32 & 2.65 \\
\hline & & 3 & 11.01 & 7.74 & 3.96 & 1.86 & 11.21 & 8.12 & 4.40 & 2.28 \\
\hline & \multirow{3}{*}{2} & 1 & 4.17 & 3.74 & 2.78 & 1.94 & 4.18 & 3.75 & 2.77 & 1.94 \\
\hline & & 2 & 8.14 & 6.46 & 3.85 & 2.14 & 8.32 & 6.79 & 4.24 & 2.62 \\
\hline & & 3 & 11.05 & 7.73 & 3.95 & 1.85 & 11.26 & 8.03 & 4.33 & 2.27 \\
\hline & \multirow{3}{*}{3} & 1 & 4.17 & 3.74 & 2.78 & 1.94 & 4.15 & 3.72 & 2.78 & 1.92 \\
\hline & & 2 & 8.14 & 6.48 & 3.84 & 2.12 & 8.27 & 6.77 & 4.36 & 2.66 \\
\hline & & 3 & 11.02 & 7.74 & 3.95 & 1.86 & 11.32 & 8.04 & 4.37 & 2.29 \\
\hline
\end{tabular}

[16] Y. Linde, A. Buzo, and R. Gray, "An algorithm for vector quantizer design," IEEE Trans. Commun., vol. 28, no. 1, pp. 84-95, 1980.

[17] N. Phamdo, F. Alajaji, and N. Farvardin, "Quantization of memoryless and Gauss-Markov sources over binary Markov channels," IEEE Trans. Commun., vol. 45, no. 6, pp. 668-675, 1997.

[18] S. Shahidi, F. Alajaji, and T. Linder, "MAP decoding of quantized sources over soft-decision fading channels with memory," in Proc. IEEE Int. Conf. Commun., 2012, pp. 2277-2282. 